\title{
System for Nature-inspired Signal Processing: Principles and Practice
}

\author{
Frank Edughom Ekpar
}

\begin{abstract}
This paper establishes the foundational principles and practice for a unified theory of arbitrary information management by disclosing systems, devices and methods for the management of substrates or biological substrates. In this context, a substrate is any aspect of any entity that is capable of responding to or emitting stimuli irrespective of whether the stimuli actually emanate from any aspect of the entity or not. Management of substrates could be achieved through the management of stimuli that modulate or moderate or influence any aspect of the substrate as well as through the management of any stimuli emanating from the substrate. The results enable a wide range of novel applications in a variety of fields with far-reaching implications. For example, the functional organization of many regions of the brain including the superior temporal cortex which is believed to play a critical role in the hierarchical processing of human visual and auditory stimuli is poorly understood. It is not known precisely which layer within which region of the brain is responsible for which aspect of visual or auditory processing. Simultaneous non-invasive acquisition of bio-signals representing contributions from multiple layers of neuronal populations within the brain could provide new insights leading to the resolution of many of these outstanding issues and provide a deeper understanding of the underlying physiological processes.
\end{abstract}

Index Terms-Nature-inspired signal processing, Noninvasive Brain-Computer Interfaces, Ampullae of Lorenzini.

\section{INTRODUCTION}

Biological systems such as plants and animals are associated with a wide variety of signals. In this context, these signals are referred to as bio-signals and are understood to include both intrinsic signals generated by the biological system for its own purposes and extrinsic signals that can be used to manipulate selected aspects of the biological system. Plants are known to communicate using chemical signals. Ian T. Baldwin and Jack C. Schultz[1] report evidence for communication between plants mediated by phenolic compounds and suggest that an airborne cue generated by damaged plant tissue may elicit biochemical changes in neighboring plants that could have an impact on the feeding and growth of phytophagous (plant-eating) insects. In essence, damaged plants seem to generate signals that are conducive to their survival as well as induce the generation of similar signals in neighboring plants. R. Karban et. al.[2] demonstrate rigorous experimental evidence for induced resistance to herbivores in wild tobacco plants following the clipping of neighboring

Published on December 23, 2019.

Frank Edughom Ekpar is with the Admiralty University of Nigeria, Nigeria.

(e-mail: frankekparng@gmail.com). sagebrush, reporting that wild tobacco plants with clipped sagebrush neighbors exhibited higher levels of the putative defensive oxidative enzyme polyphenol and experienced significantly reduced leaf damage from grasshoppers and cutworms than controls. Echo-locating bats have been reported to use sonar for navigation and foraging[3-7]. Sharks and related species of fish use electro-sensory structures known as ampullae of Lorenzini for the localization of prey[8-12]. The artificial pacemaker and defibrillator are well known examples of the application of electrophysiological signals associated with the human heart[13-17]. Ultimately, in many biological systems found in nature - including the human body -, bio-signals are generated in, mediated by, and exert their influence on intracellular and/or extracellular (in the case of multicellular systems) structures and processes. In this regard, many studies of the electrophysiological and related characteristics of intracellular and extracellular structures and tissues have been reported in the literature and include the Hodgkin-Huxley model that gives a theoretical description of excitable membrane. The patch clamp method was introduced by Edwin Neher and Bert Sakmann and permitted the measurement of the membrane current of a single ion channel. Further refinement of the patch clamp technique allowed the determination of cell membrane capacitance and subsequently led to studies harnessing minute changes in membrane surface area to characterize secretory processes[18-31].

A wide variety of modalities are harnessed in the study of bio-signals associated with the human brain. These include, but are not limited to, positron emission tomography (PET)[32], single-photon emission computed tomography (SPECT), electroencephalography (EEG), electrocorticography (EcoG), magneto-encephalography (MEG), functional magnetic resonance imaging (fMRI) and functional near-infrared spectroscopy (fNIR). Each of these modalities has its merits and demerits when compared with the others. Electroencephalograph (EEG) and magnetoencephalography (MEG) are remarkable in the sense that the EEG signal originates from the electrical activity of neuronal populations and can be measured directly using simple electrodes. Similarly, based on Maxwell's equations, the electrical activity of neuronal populations yields a magnetic field that can be measured directly using a magnetometer in MEG[33, 34]. This is not the case for other modalities that rely on indirect measures of brain activity. Modern MEG devices typically utilize ultrasensitive superconducting quantum interference devices (SQUIDs)[35] arrays for the detection of the weak magnetic fields that originate from the brain's electrical activity. The EEG has is a non-invasive technique (involving the placement of electrodes or sensors on the scalp), can be implemented at relatively low cost, imposes fewer restraints 

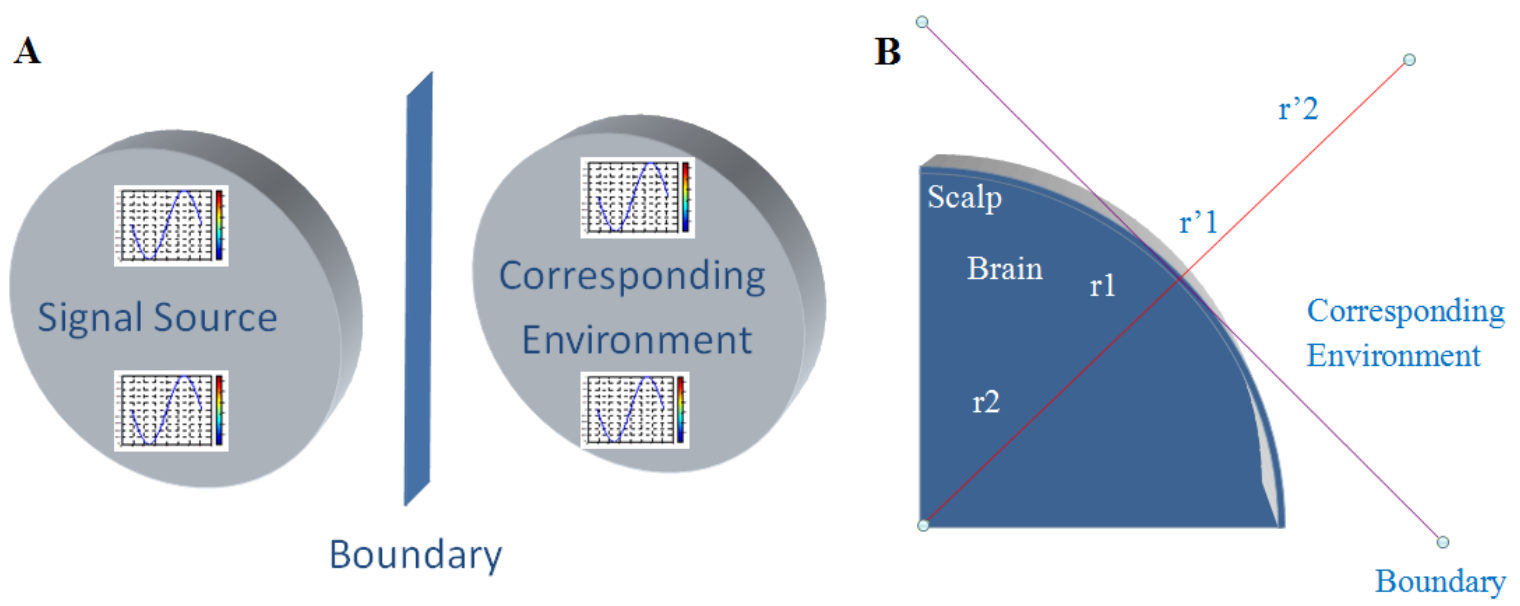

Fig. 1A. Outline of model. B. Distances from the boundary in a radial direction from the scalp.

of 1 millisecond - but is hampered by low spatial resolution. The optimum electrode distance for the EEG seems to be between $10.0 \mathrm{~mm}$ and $50.0 \mathrm{~mm}$ on the basis of estimated variable brain-skull-scalp resistivity ratios and the use of the reciprocity theorem, superposition principle, lead field theorem and theoretical spatial frequency (spatial Nyquist) considerations[36-41]. Attempts to localize the sources of the EEG signals (or to solve the forward and inverse problems) typically employ large numbers of electrodes (in the order of 100 electrodes) and provide only crude estimates[42-44]. Researchers have demonstrated the feasibility of brain-computer interfaces (BCIs) based solely on noninvasively acquired EEG signals[45]. Generally, contemporary noninvasive EEG measurement systems utilize electrodes placed on the scalp with a single electrode or senor per site. Thus for each site on the scalp, only a single signal stream - presumably representing the superposition of signal contributions of layers of neuronal populations beneath the site - is typically acquired. However, the simultaneous acquisition of distinct signals representing the contributions of different layers of neuronal populations by multiple electrodes at the same site could significantly improve the spatial resolution of the EEG, provide new insights into the underlying physiological processes and open up new avenues for the application of the EEG.

Section I has introduced this paper and provided a survey of the relevant literature. The rest of this paper is organized as follows. Section II introduces the problem definition, methodology/approach in the form of the model employed for arbitrary data management. In Section III, a bio-signal acquisition system inspired by Ampullae of Lorenzini is presented. Section IV describes the data acquisition setup. Results are presented in Section V. In Section VI, a discussion of the results is presented while Section VII contains concluding remarks and future scope while Section VIII contains references.

\section{Problem Definition/Methodology/ApProACH: MODEL}

In this model, approximations to carefully selected representative features of the source of the bio-signals are utilized for characterization and manipulation of the underlying biological system. This is illustrated in Fig. 1A which depicts an outline of the model. An environment corresponding to selected features of the source is created and a strategy that utilizes the corresponding environment for the characterization and/or manipulation of the underlying biological system is pursued.

The choice of features to approximate in the corresponding environment depends on the application of the model. Similarly, the characteristics of the boundary between the source of the bio-signals and the corresponding environment created in the model depend on the features of the source and the corresponding environment.

Consider the situation where the source of the bio-signals is the human brain and the goal is the acquisition of the corresponding electroencephalography (EEG) signals with minimal or no corruption from the distortions introduced by the brain tissue, skull and scalp. Then based on this model, an environment could be created outside the brain - with a boundary comprising a conducting medium in contact with the scalp and the corresponding environment - in which approximations to the distortions are used to inform the placement of sensors in such a manner as to mitigate or eliminate the effects of the distortions. Approaches such as spatial de-convolution could be used to correct distortions in a post-acquisition step[46] but it would be more convenient to mitigate the effects of such distortions at the signal acquisition stage. Now suppose the goal is to simultaneously acquire the EEG from different depths within the brain. In this case an environment - outside the brain - that approximates the propagation delays as the signals propagate from sources within the brain to the scalp could 
be created and sensors could be positioned in the corresponding environment in a manner that would allow the simultaneous acquisition of signals from different depths within the brain. Here the boundary could also comprise a conducting medium in contact with the scalp and the corresponding environment. The following predictions can be made based on this model:

PREDICTION I: Since signals from sources at locations deeper in the brain are likely to reach the scalp later than signals originating from neuronal populations or brain regions closer to the scalp, sensors in the corresponding environment closer to the boundary (in a radial direction from the scalp) are likely to detect signals in which contributions from neuronal populations at deeper locations within the brain predominate. Conversely, sensors located farther from the boundary are likely to detect signals in which the contributions of neuronal populations that are closer to the boundary predominate. Referring to the illustration in Figure 1B, a sensor located at r'1 is likely to detect signals in which the contributions of neuronal populations closer to $\mathrm{r} 2$ predominate while a sensor located at r'2 is likely to detect signals in which the contributions of neuronal populations closer to $\mathrm{r} 1$ predominate.

PREDICTION II: Sensors placed over the same site but separated from each other (in a radial direction from the scalp, such as sensors located at r' 1 and r'2 in Figure 1B) are likely to detect signals in which contributions from different levels within the brain predominate.

PREDICTION III: Sensors embedded directly at different levels within the brain should detect signals similar to those detected by sensors located at corresponding positions within the corresponding environment.

The first prediction (Prediction I) can be justified on the basis of the laws of electromagnetic wave propagation as the signals responsible for the EEG are electrical in nature and induce a corresponding magnetic field.

One way to verify Prediction III would be to surgically implant sensors at different depths within the brain and compare the signals acquired with the signals acquired at corresponding locations within the corresponding environment. This would be an invasive procedure.

Prediction II can be verified by demonstrating the acquisition of distinct signals from two sensors located at the same site on the scalp. The ampullae of Lorenziniinspired bio-signal acquisition system described later in this paper is used to provide just such a demonstration.

\section{AMPULlAE OF LORENZINI-INSPIRED BIO-SIGNAL ACQUISITION SYSTEM}

This section introduces a bio-signal acquisition system inspired by ampullae of Lorenzini and based on the model described here that harnesses approximations to propagation delays as signals traverse the source to reach the boundary to facilitate the simultaneous acquisition of signals from different layers within the source. The bio-signal acquisition system comprises a grid of sensor ensembles, each sensor ensemble comprising a collection of sensors disposed on an arbitrarily shaped $\mathrm{N}$-dimensional $(\mathrm{N}=1,2,3$, etc) surface with each sensor in contact with a suitable medium (conducting medium for applications such as EEG) which in turn is in contact with a surface associated with the source of the bio-signals.

Ampullae of Lorenzini are electroreceptive units in elasmobranchs comprising jelly-filled canals found on the head of the animal which form a system of sense organs, each of which receives stimuli from the outside environment through the dermis and epidermis. Each canal ends in groups of small bulges lined by the sensory epithelium. A small bundle of afferent nerve fibers innervates each ampulla. Although the lengths of the canals vary from species to species (even within any one fish), the pattern of distribution is approximately species specific[8-12].

Figure $2 \mathrm{~A}$ depicts a representative sensor ensemble with four separate co-planar electrodes labeled E1, E2, E3 and E4 and contained in a plastic (non-conducting) cylindrical casing with an inner diameter (d) of $10.0 \mathrm{~mm}$ and a height (h) of $37.0 \mathrm{~mm}$. The cylinder was filled with a saline $(\mathrm{NaCl})$-soaked sponge medium and each of the electrodes was a stainless steel wire with a diameter of $1.1 \mathrm{~mm}$. The inter-electrode distance (id) was $5.0 \mathrm{~mm}$ and the same for all four electrodes while the distance between the first or base electrode E1 and the boundary between the sensor ensemble and the signal source surface or scalp (ed) was $7.0 \mathrm{~mm}$. For EEG data acquisition, each electrode in the ensemble was connected to one electrode - replacing the original sensor on a wireless Emotiv EPOC headset containing 14 electrodes positioned in accordance with the international 10-20 system as shown in Figure 2B.

E1 was connected to AF3, E2 to AF4, E3 to F3 and E4 to F4. P7 and P8 served as references and were attached to the original sensors supplied with the Emotiv EPOC headset. The Emotiv EPOC headset is supplied with a software tool named TestBench that can be used to record and store EEG data and displays an electrode contact quality map on which green indicates good contact quality, black indicates no contact, red indicates very poor contact, orange indicates poor contact, yellow indicates fair contact - and is the source of the image on which Figure 2B is based.

Canals in the ampullae could correspond to the conducting medium in Figure 2A. The sensor casing, housing or support could correspond to the sensorcontaining basal region or alveoli of the ampullae. The walls of the sensor-containing basal region or alveoli of the ampullae are typically composed of high resistive or nonconducting material - as is the wall of the sensor casing in Figure 2A. As is generally the case with naturally-occurring ampullae, each ampulla contains a plurality of sensors that could be arranged in an omni-directional fashion for optimum signal coverage. It should be noted that arbitrary configurations of sensor elements are permitted by this model so such omni-directional sensor topographies could also be employed where practicable. The jelly or hydrogel that fills the canals found in ampullae could correspond to the conducting medium - a saline $(\mathrm{NaCl})$-soaked sponge in this case. As with the sensor topology, the choice of medium depends on the requirements of the specific application of the model. United States Patent Number 7567274 describes a versatile image acquisition device comprising at least one grid of one or more focusing elements disposed on an $\mathrm{N}$ - 


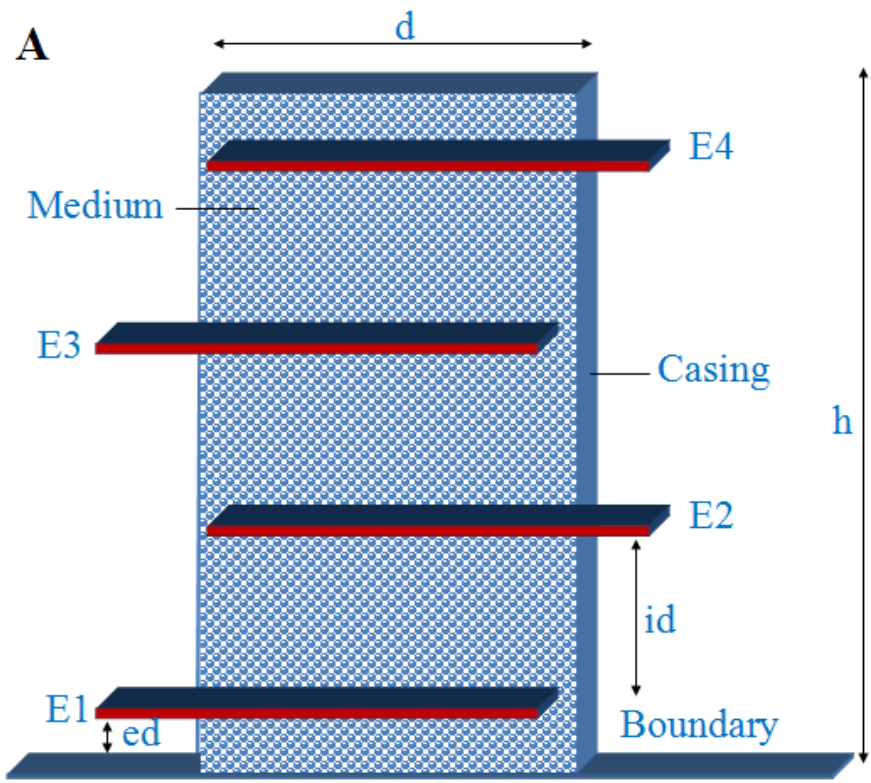

B

\section{Signal Source}

Figure 2: A: Vertical cross-section through a representative sensor ensemble. B: Electrode positions on the Emotiv EPOC Headset.

dimensional and arbitrarily shaped surface, at least one grid of one or more sensor elements disposed on an Ndimensional and arbitrarily shaped surface, and optionally, at least one grid of one or more stimulus guide elements disposed on an $\mathrm{N}$-dimensional and arbitrarily shaped surface, where $\mathrm{N}$ can be chosen to be $1,2,3$, or any other suitable quantity[47]. The grid of sensor ensembles illustrated here defer from the sets of equivalent methods, systems and devices described in the ' 274 patent in that the sensor ensembles described here are simpler and lack the focusing elements of the ' 274 patent.

\section{DATA ACQUiSITION AND ANALYSIS}

\section{IV.1 Data Acquisition}

With the electrodes E1, E2, E3 and E4 replacing the original sensors at locations AF3, AF4, F3 and F4 and the reference electrodes at locations $\mathrm{P} 7$ and $\mathrm{P} 8$ attached to the original sensors, EEG data was acquired by placing the sensor ensemble illustrated in Figure 2A on a subject's scalp at a location close to F4 on the Emotiv EPOC headset. The TestBench software supplied with the headset was used to record and store the data and ultimately convert it to text format.

\section{IV.2 Configuration Data Analysis}

What effect, if any, does the topography of the transducer ensemble have on the distinctness of the EEG or other data associated with a biological substrate recorded or generated by each of the transducers in the ensemble?

To investigate this question in the case where the transducers are configured as sensors or electrodes, the acquired EEG data could be analyzed using measures of the "electrical distance" between electrodes, the Pearson product-moment correlation coefficients between pairs of EEG signal streams corresponding to pairs of electrodes and scatter plots for electrode pairs while being cognizant of the actual spatial distances between the electrodes.

\section{IV.2.1 Computing Correlation Coefficients}

For any pair of electrodes, the Pearson product-moment correlation coefficient (r) can be calculated thus:

$$
r=\frac{\sum_{i=1}^{n}\left(X_{i}-\bar{X}\right)\left(Y_{i}-\bar{Y}\right)}{\sqrt{\sum_{i=1}^{n}\left(X_{i}-\bar{X}\right)^{2} \sqrt{\sum_{i=1}^{n}\left(Y_{i}-\bar{Y}\right)^{2}}}}
$$

where $\bar{X}^{\text {denotes the sample mean for EEG or any other }}$ suitable type of data recorded at the first electrode, $\bar{Y}$ is the sample mean for EEG or any other suitable type of data recorded at the second electrode, and $\mathrm{n}$ is the number of samples.

\section{IV.2.2 Measuring Electrical Distance Using the Hjoth Laplacian}

A linear approximation to the surface Laplacian can be computed using the Hjorth algorithm [51]. In the Hjorth waveform $H_{i}(\mathrm{t}, \mathrm{N})$, the contribution to the signal from each electrode $i$ is expressed as the difference between the 
time-varying potential $P_{i}(t)$ and the scaled sum of the potentials $P_{j}(t)$ at each of $\mathrm{N}$ neighboring electrodes, as expressed in the following equation:

$$
H_{i}(t, N)=P_{i}(t)-\sum_{j=1}^{N} P_{j}(t) W_{i-j}(N)
$$

, where $W_{i-j}$ is a weighting factor for each neighbor that is inversely proportional to the distance $d_{i-j}$ between the electrodes.

$$
W_{i-j}(N)=\frac{1 / d_{i-j}}{\sum_{k=1}^{N} 1 / d_{i-k}}
$$

In the intrinsic Hjorth algorithm, the spatial distance $\boldsymbol{d}_{i-j}$ is replaced by the non-spatial "electrical distance" $D_{i-j}$ reflecting the electrical similarity between electrodes $i$ and $j$. The potential difference waveform $P_{i-j}(t)$ between two electrodes $i$ and $j$ is given by:

$$
P_{i-j}(t)=P_{i}(t)-P_{j}(t)
$$

The "electrical distance" can then be computed as:

$$
D_{i-j}=\frac{1}{T} \sum_{t=1}^{T}\left(P_{i-j}(t)-\overline{P_{i-j}(t)}\right)^{2}
$$

In foregoing equation for the computation of the "electrical distance", $\overline{P_{i-j}(t)}$ is the mean potential difference waveform. This gives the temporal variance of the difference potential waveform.

According to [52], replacing the spatial distance $\boldsymbol{d}_{i-j}$ with the temporal variance of the difference potential waveform $D_{i-j}$ yields the intrinsic Hjorth transform in the case of a single neighbor. In order to detect electrolyte bridges between electrodes, it is sufficient to limit consideration of electrical distances to the detection of the single nearest neighbor. This is equivalent to setting $N=1$ in the equation describing the linear approximation to the surface Laplacian that can be computed using the Hjorth algorithm [52].

\section{RESULTS}

\section{V.1 Representative Sensor Ensemble (Fig. 2a)}

Approximately 3 minutes of spontaneous EEG data was acquired from a subject at rest using the setup described previously. Pearson product-moment correlation coefficients were calculated and scatter plots were generated for the E1-E2, E1-E3 and E1-E4 electrode pairs. Table 1 displays the correlation coefficient and interelectrode distance (measured from E1), Figure 3A graphically depicts the relationship between the interelectrode distance and correlation coefficient while Figures 3B-3D show the scatter plots for the E1-E2 E1-E3 and E1-

\begin{tabular}{|c|c|c|c|}
\hline Electrode & 2 & 3 & 4 \\
\hline $\begin{array}{ll} & \text { Correlation } \\
\text { Coefficient } & \\
\end{array}$ & .9794 & .9521 & .8914 \\
\hline Distance $(\mathrm{mm})$ Interelectrode & .0 & 0.0 & 5.0 \\
\hline
\end{tabular}
E4 electrode pairs.

Table 1: Correlation coefficients and interelectrode distances for the E1-E2 E1-E3 and E1-E4 electrode pairs.

\section{V.2 Alternative Sensor Configuration}

An alternative configuration in which two sensors were placed orthogonally with a separation of $10.0 \mathrm{~mm}$ along the axis of the cylinder was used to mitigate the putative effects of placing electrodes on the same plane along the axis and directly above each other as shown in Figure 2A. This alternative configuration is shown in Figure 4A where the inner diameter of the containing plastic cylinder D is 10.0 $\mathrm{mm}$, its height $\mathrm{L}$ is $20.0 \mathrm{~mm}$, the height of the first electrode (SE1) - HSE1 is $2.0 \mathrm{~mm}$ and the height of the 
A
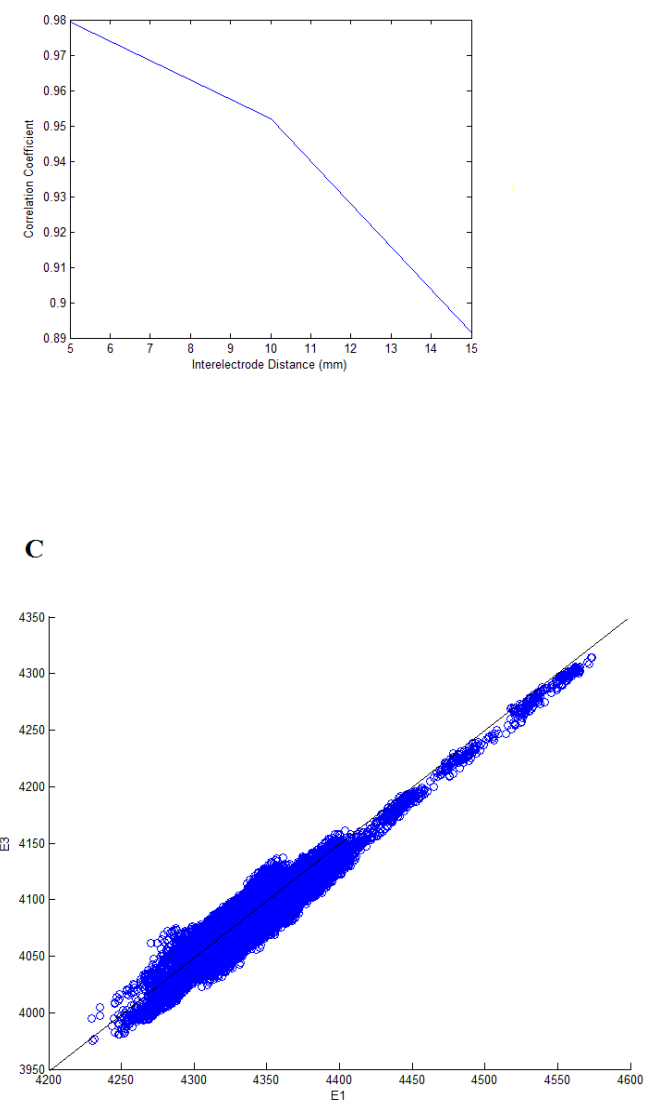

B

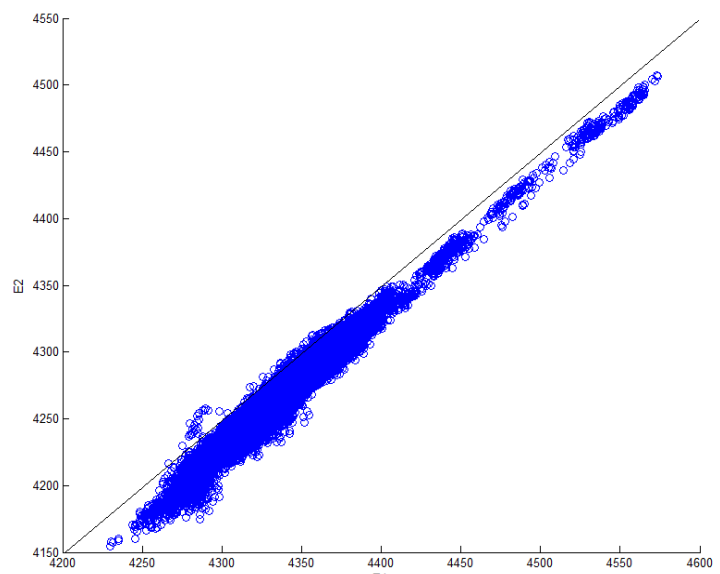

D

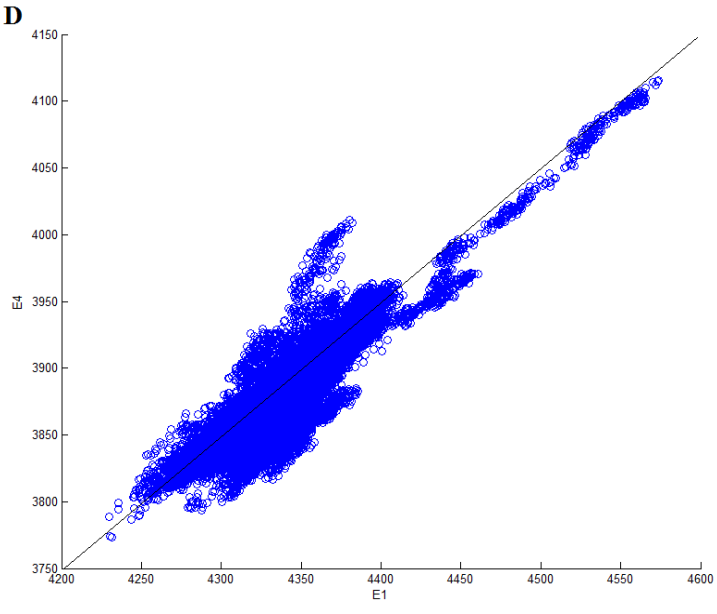

Figure 3A. Relationship between inter-electrode distance and correlation coefficient. B. Scatter plot of EEG data for E1-E2 electrode pair. Correlation coefficient, $r=0.9794$. C. Scatter plot of EEG data for E1-E3 electrode pair. Correlation coefficient, $r=0.9521$. D. Scatter plot of EEG data for E1-E4 electrode pair. Correlation coefficient, $\mathrm{r}=0.8914$.

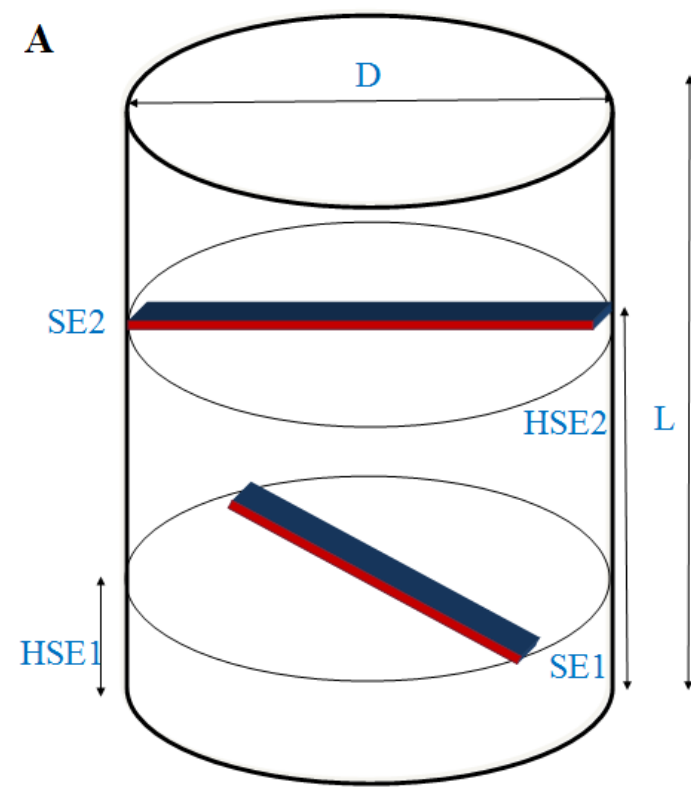

$\mathbf{B}$

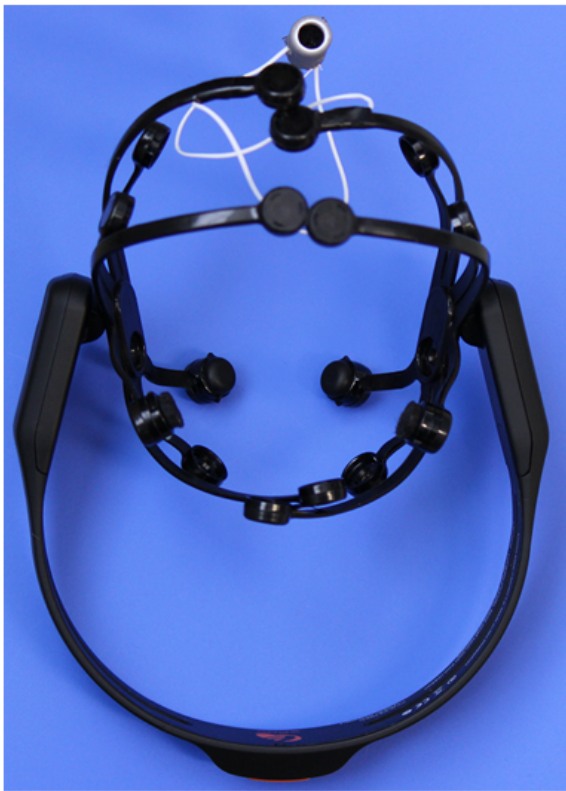

Figure 4A. Alternative sensor ensemble. B. Experimental setup with alternative sensor ensemble. 
$\mathbf{A}$

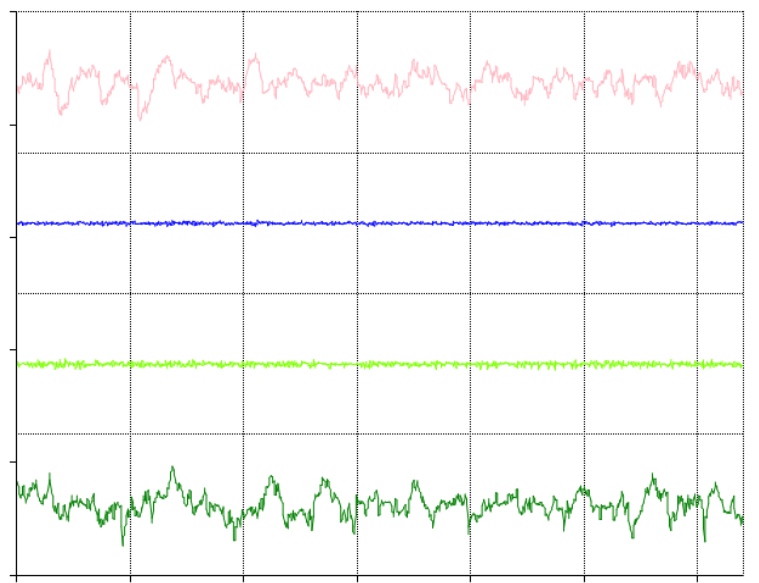

B

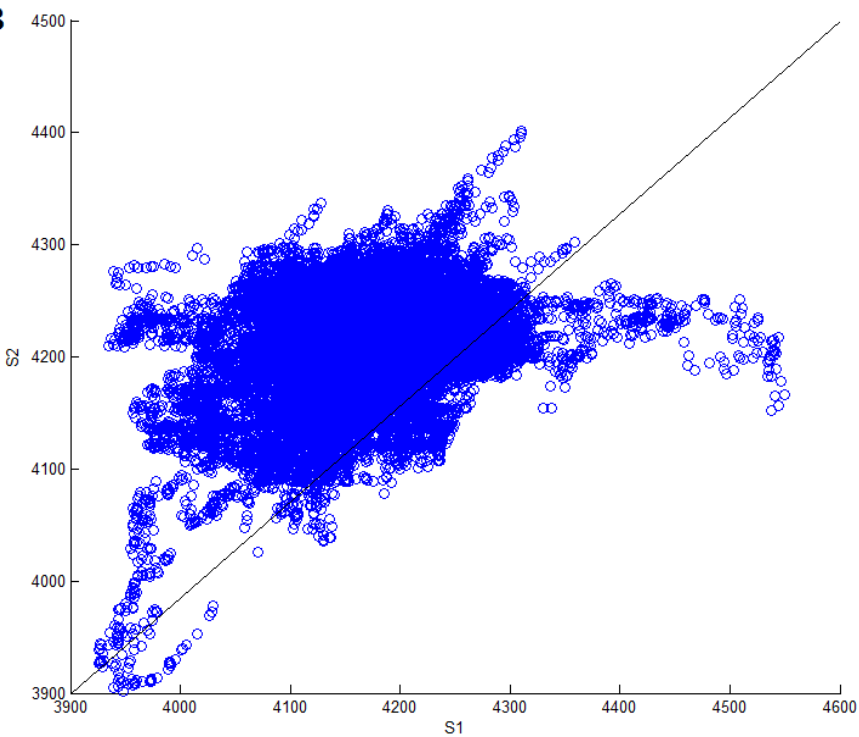

Figure 5A. TestBench screenshot showing SE1-SE2 EEG data. The top and bottom brainwaves correspond to data acquired at SE1 (AF3) and SE2 (AF4), respectively. Data from the two reference locations (P7 and P8) appear in the middle of the screenshot. B. Scatter plot of EEG data for SE1-SE2 electrode pair. Correlation coefficient, $r=0.3281$

second electrode (SE2) - HSE2 is $12.0 \mathrm{~mm}$ - giving an inter-electrode distance of $10.0 \mathrm{~mm}$. Figure $4 \mathrm{~B}$ depicts a photograph of the arrangement. Using this alternative configuration with the sensor ensemble located near AF4 on the subject's scalp, approximately 3 minutes of spontaneous EEG data was acquired from a subject at rest using the Emotiv EPOC headset with the electrodes at AF3 and AF4 attached to SE1 and SE2, respectively, while the reference electrodes were attached to the original sensors. Figure $5 \mathrm{~A}$ is a screenshot of a portion of the resulting EEG data displayed in TestBench. The scatter plot of the data acquired at the SE1 and SE2 sensor site appears in Figure 5B. The correlation coefficient, $r$, was 0.3281 .

\section{V.3 Further Alternative Sensor Configurations}

Furthermore, consider the simple but effective sensor ensemble topography contained within a cylinder that partitions the cylinder into $\mathrm{n}$ (where $\mathrm{n}$ is a positive integer) unique planes passing through the axis of the cylinder with each plane separated by an angle of $\pi / \mathrm{n}$ radians from each of its nearest neighbors.

The edges of sensors or actuators (collectively transducers) in a configuration in which a single sensor or actuator or transducer is embedded in each plane of the cylinder - at a distinct height from the base - circumscribe a double helix.

One of ordinary skill in the field would appreciate that simple techniques such as the computation of the Pearson product-moment correlation coefficients, the display and inspection of scatter plots and the computation of the electrical distances between selected electrodes or sensors or actuators or transducers as well as any other suitable techniques would facilitate the comparison of the performance of this double helix topography with the referential arrangement in which sensors or actuators or transducers are stacked one above the other on a plane intersecting the axis of the cylinder.

\section{V.4 Cylinder Partitioning}

The goal is to minimize the effects of inter-electrode interference in sensor or actuator or transducer ensembles comprising multiple electrodes or sensors or actuators or transducers. In the referential topography, sensors or electrodes or actuators or transducers could be stacked one above the other in the sensor or electrode or actuator or transducer ensemble. Experimental results suggest that limiting overlap between neighboring sensors or electrodes or actuators or transducers could mitigate the effects of inter-electrode interference and lead to the acquisition (or generation) of more distinct signal streams with greater information content. Accordingly, the partitioning of the containing cylinder into unique planes passing through the axis of the cylinder is proposed. Sensors or electrodes or actuators or transducers can then be embedded in each unique plane at a distinct height from the base of the cylinder.

Figure 6 shows a top view of a containing cylinder outlining two representative planes $\mathrm{P} 1$ and $\mathrm{P} 2$. The angle between the planes is $\theta$. In Figure 7, the same arrangement is depicted as viewed from the side in three dimensions. The number of planes, $\mathrm{n}$, is arbitrary in principle. 


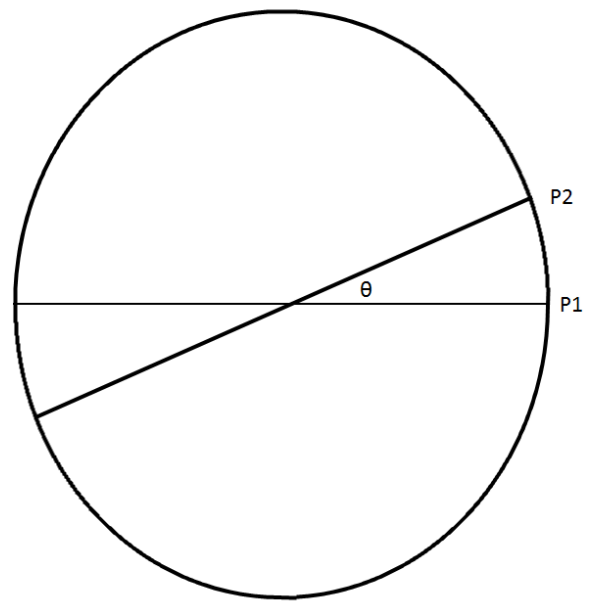

Figure 6. Top view of a containing cylinder outlining two representative planes $\mathrm{P} 1$ and $\mathrm{P} 2$.

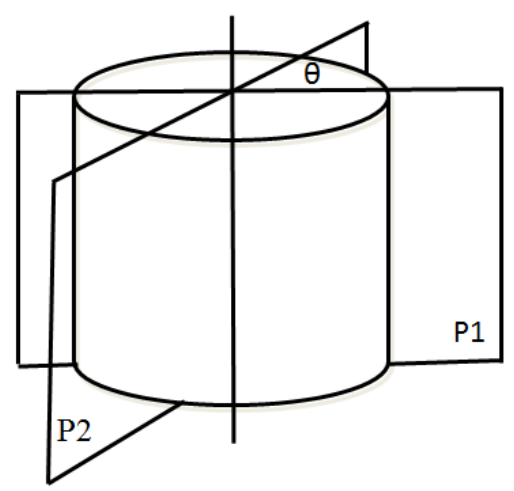

Figure 7. Side view of a containing cylinder outlining two representative planes $\mathrm{P} 1$ and $\mathrm{P} 2$.

The following formula gives the inter-plane angle, $\theta$, in terms of the number of planes, $n$.

$$
\theta=\frac{\pi}{n}
$$

, where $\theta$ is the inter-plane angle in radians and $n$ is the number of planes.

An arbitrary number of transducers, m, can be embedded within each unique plane. This can be done by subdividing the plane into $\mathrm{m}$ separate strips and embedding a transducer in each strip as shown in Figure 8 where transducers e1, e2, .., em are embedded in the strips, one transducer per strip.

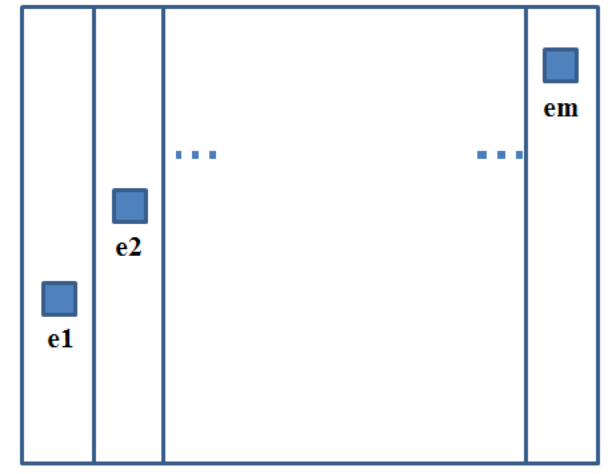

Figure 8. $\mathrm{m}$ transducers embedded within a plane divided into $\mathrm{m}$ separate strips.
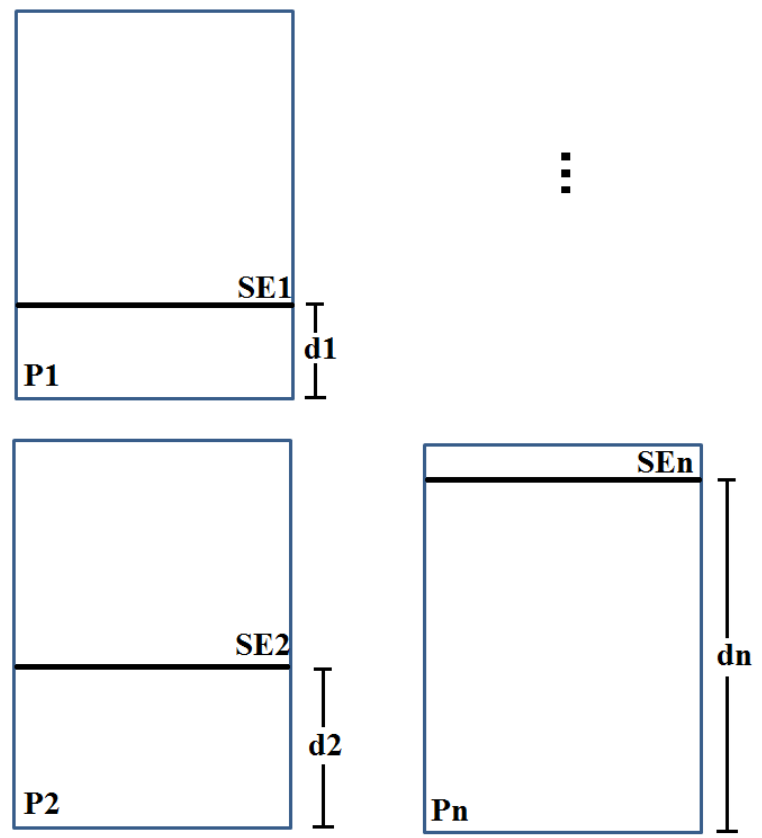

Figure 9. Configuration with a single transducer embedded in each of $n$ separate planes at a distinct height from the base of the cylinder.

In practice, both the number of planes $\mathrm{n}$ and the number of strips per plane $\mathrm{m}$ are limited by the physical characteristics (principally the sizes) of the cylinder and transducers.

For simplicity, just one transducer could be embedded per plane with each transducer at a distinct height from the base of the cylinder. This arrangement is shown in Figure 9 in which the planes are labeled P1, P1, .., Pn and the corresponding transducers are labeled SE1, SE2,..,SEn. Each transducer is located at a distinct height (depicted as $\mathrm{d} 1, \mathrm{~d} 2, \ldots, \mathrm{dn}$ ) from the base of the cylinder. It is instructive to note that since each unique plane intersects the axis of the cylinder and contains a single transducer, the sensors overlap around the axis of the cylinder

The regions of the transducers intersecting the surface of the cylinder in this simple configuration (one transducer per plane at a distinct height from the base of the cylinder) circumscribe a double helix as illustrated in Figure 10. 


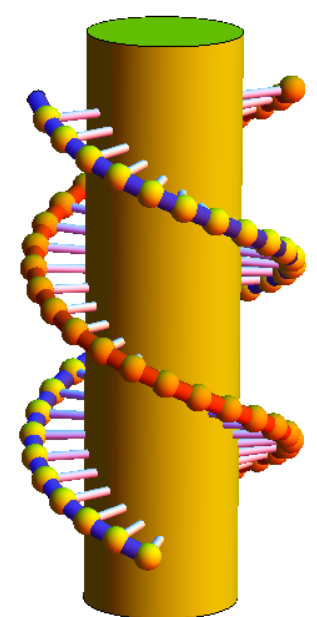

Figure 10. Double helical structure resulting from the configuration with a single transducer embedded in each of $n$ separate planes at a distinct height from the base of the cylinder.

As noted previously, the double helical configuration with a single transducer per plane intersecting the axis of the cylinder leads to the overlap of transducers around the axis. This overlap can be removed by splitting each transducer into two transducers (one on each helix) with some space between them - resulting in the double-stranded helical topography depicted in Figure. 11.

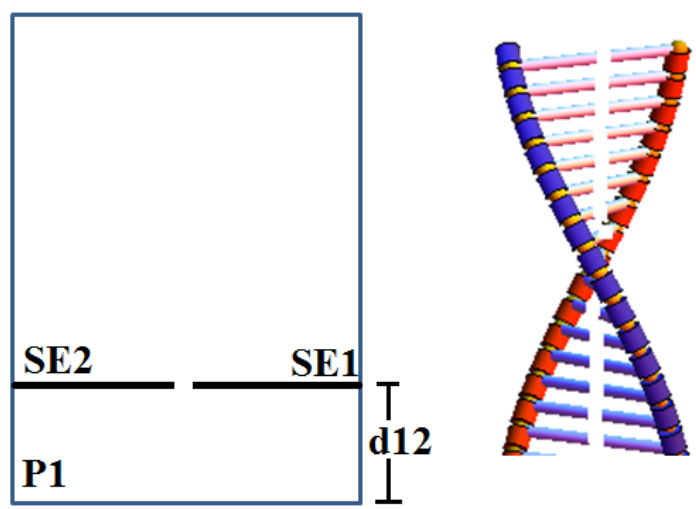

Figure 11. Splitting of a transducer on a plane P1 into two opposing transducers SE1 and SE2 at a height d12 from the base of the cylinder and the double-stranded double helix formed by partitioning the containing cylinder into unique planes and embedding two opposing and horizontally separated transducers per plane at a distinct height from the base of the cylinder.

\section{DISCUSSION}

The apparent presence of electrolyte bridges in the sensor ensembles should preclude the measurement of distinct signal streams[48, 49] and should yield correlation coefficients higher than 0.99. As already noted, contemporary EEG systems typically acquire a single signal stream per site and are configured in a manner that virtually precludes the simultaneous acquisition of multiple signal streams per site. Furthermore, since the tangential separation of the electrodes in the configurations presented in this paper is much smaller than $10.0 \mathrm{~mm}$, tangential contributions should be substantially identical ${ }^{36-41}$ with a correlation coefficient of 1.0. However, as illustrated by the results presented, distinct signal streams can indeed be obtained from sensors placed at the same site on the surface representing the source of the bio-signals. The signals acquired are likely to originate from sources located at different depths (in a radial direction) and reach the sensors at different times due to propagation delays in the intervening media. Although the Emotiv EPOC is a consumer-grade system featuring a single analogue-todigital converter and hence only approximates parallel sampling of the electrodes, it is sufficient to simultaneously acquire distinct signals from sensors placed at the same site. The results indicate that the correlation between signals tends to decrease as the inter-electrode distance increases in the radial direction. Placing the electrodes orthogonally as shown in Fig. 4A-B resulted in a significant decrease in the correlation coefficient and made it possible to notice differences between the signals via mere visual inspection suggesting that this could improve the amount of additional independent information that can be acquired at the same site using this arrangement.

\section{CONCLuSion AND Future ScOPE}

We have established the foundational principles and practice for a unified theory of arbitrary information management by disclosing systems for the simultaneous non-invasive acquisition of distinct bio-signals from different layers or depths within the bio-signal source at a single sensor site. Using invasive cortical implants, researchers obtained direct evidence for acoustic-to-higher order phonetic level encoding of speech sounds in human language receptive cortex[50]. However, similar or better results - including the precise specification of which layer within the cortex is implicated in which aspect of speech sound processing could be obtained non-invasively using our bio-signal acquisition system. Application of our results could provide new insights into the functional organization of the brain and other biological systems in which signals at different layers contribute to system behavior. Our system enables a wide range of clinical and non-clinical application domains.

\section{REFERENCES}

[1] Baldwin, I. T. \& Schultz, J. C., "Rapid Changes in Tree Leaf Chemistry Induced by Damage: Evidence for Communication Between Plants," Science 221, 277-279 (1983).

[2] Karban, R et. al., "Communication Between Plants: induced resistance in wild tobacco plants following clipping of neighboring sagebrush," Oecologia 125, 66-71 (Springer-Verlag 2000).

[3] Pye, J. D., "A theory of echolocation by bats," J. Laryng. Otol. 74, 718-729 (1960).

[4] Pye, J. D., "Perception of distance in animal echolocation," Nature Lond. 190, 362-363 (1961).

[5] Pye, J. D., “ A. Correlated orientation sounds and ear movements of horseshoe bats," Nature Lond. 196, 1185-1188 (1963).

[6] Moss, C. F., "Acoustic information available to bats using frequency modulated sounds for the perception of prey," J. Acoust. Soc. Am. 95, 2745-2756 (1994).

[7] Jensen, M. E., "Detection of Prey in s Cluttered Environment by the Northern Bat Eptesicus Nilssonii," J. Exp. Biol. 204, 199-208 (2001). 
[8] Murray, R. W., "The Response of Ampullae of Lorenzini of Elasmobranchs to Electrical Stimulation,” J. Exp. Biol. 39, 119-128 (1962).

[9] Murray, R. W., "Electrical Sensitivity of the Ampullæ of Lorenzini," Nature 187, 957 (1960).

[10] Kalmijn, A. J., "Electro-perception in Sharks and Rays," Nature 212, 1232-1233 (1966).

[11] Kalmijn, A. J., "Electric and magnetic field detection in elasmobranch fishes," Science 26, 916-918 (1982).

[12] Brown, B. R., "Modeling an electro-sensory landscape: behavioral and morphological optimization in elasmobranch prey capture," $J$. Exp. Biol. 205, 999-1007 (2002).

[13] Jenkins, M. W. et. al., "Optical pacing of the embryonic heart," Nature Photonics 4, 623-626 (2010).

[14] Furnan, S. \& Schwedel, J. B., "An Intracardiac Pacemaker for Stokes-Adams Seizures," Pac. Clin. Electrophysiol. 29, 453-458 (2006)

[15] Bohm, A et. al., "Clinical Observations with Long-term Atrial Pacing," Pac. Clin. Electrophysiol. 21, 246-249 (1998).

[16] Halperin, D. et. al., "Pacemakers and Implantable Cardiac Defibrillators: Software Radio Attacks and Zero-Power Defenses," IEEE Symp. Sec. Priv. 129-142 (2008).

[17] Yatani, A. et. al., "Heart rate regulation by $\mathrm{G}$ proteins acting on the cardiac pacemaker channel," Science 249, 1163-1166 (1990).

[18] Hoshi, T., Zagotta, W. N. \& Aldrich, R. W., "Biophysical and molecular mechanisms of shaker potassium channel inactivation," Science 250, 533-538 (1990).

[19] Hodgkin, A. L. \& Huxley, A. F., "A quantitative description of membrane current and its application to conduction and excitation in nerve," J. Physiol. (Lond.) 117, 500-544 (1952).

[20] Hodgkin, A. L., Huxley, A. F. \& Katz, B., "Measurement of currentvoltage relations in the membrane of the giant axon of Loligo," $J$. Physiol. (Lond.) 116, 424-448 (1952).

[21] Noble, D., "Application of Hodgkin-Huxley equations to excitable tissues," Physiol. Rev. 46, 1-50 (1966).

[22] Neher, E., Sakmann, B. \& Katz, B., "Single-channel currents recorded from membrane of denervated frog muscle fibers," Nature 260, 799-802 (1976)

[23] Neher, E., Sakmann, B. \& Katz, B., "The patch clamp technique," Sci. Am. 266, 28-35 (1992).

[24] Hamill, O. P. et. al., "Improved patch clamp techniques for high resolution current recording from cells and cell-free membranes," Pflüger Arch. ges. Physiol. 391, 85-100 (1981).

[25] Sakmann, B. \& Neher, E., "Patch clamp techniques for studying ionic channels in excitable membrane," Annu. Rev. Physiol. 46, 455472 (1984).

[26] Cole, K. S. \& Moore, J. W., "Potassium ion current in the squid giant axon: Dynamic characteristics," Biophys. J. 1, 1-14 (1960).

[27] Armstrong, C. W. \& Hille, B., "The inner quaternary ammonium ion receptor in potassium channels of the node of Ranvier," J. Gen. Physiol. 59, 388-400 (1972).

[28] Neher, E. \& Marty, A., "Discrete changes of cell membrane capacitance observed under conditions of enhanced secretion in bovine adrenal chromaffin cells," Proc. Nat. Acad. Sci. USA 79, 6712- 6716 (1982).

[29] Unwin, P. N. T. \& Zampighi, G., "Structure of the junctions between communicating cells," Nature 283, 545-549 (1980).

[30] Toyoshima, C. \& Unwin, N., "Ion channel of acetylcholine receptor reconstructed from images of postsynaptic membranes," Nature 336, 247-250 (1988).

[31] Patlak, J. B. \& Ortiz, M., "Two modes of gating during late $\mathrm{Na}+$ channel currents in frog sartorius muscle," J. Gen. Physiol. 87, 305326 (1986).

[32] Phelps, M. E. \& Mazziotta, J. C., "Positron emission tomography: human brain function and biochemistry," Science 228, 799-809 (1985).

[33] Cohen, D., Edelsack, E. A. \& Zimmerman, J. E., "Magnetocardiograms taken inside a shielded room with a superconducting point contact magnetometer," Appl. Phys. Lett. 16, 278-280 (1970).

[34] Cohen, D., "Magnetoencephalography: detection of the brain's electrical activity with a superconducting magnetometer," Science 175, 664-666 (1972).

[35] Zimmerman, J. E., Theine, P. \& Harding, J. T., "Design and operation of stable rf-biased superconducting point-contact quantum devices, etc.," Appl. Phys. Lett. 41, 1572-1580 (1970).

[36] Malmivuo, J., Suihko, V. \& Eskola, H., "Sensitivity distributions of EEG and MEG measurements," IEEE Trans. Biomed. Eng. 44, 196208 (1997).
[37] Oostendorp, T. F., Delbecke, J. \& Stegman, D. F., "The conductivity of the human skull: Results in vivo and in vitro measurements," IEEE Trans. Biomed. Eng. 47, 1487-1492 (2000).

[38] Rush, S. \& Driscoll, D. A., "EEG-electrode sensitivity-An application of reciprocity," IEEE Trans. Biomed. Eng. 16, 15-22 (1969).

[39] Junhofer, M. et. al., "Statistical control of artifacts in dense array EEG/MEG studies,” Psychophysiology 37, 523-532 (2000).

[40] Freeman, W. J. et. al., "Spatial spectra of scalp EEG and EMG from awake humans," Clin. Neurophysiol. 114, 1053-1068 (2003)

[41] Väisänen, O. \& Malmivuo, J., "Improving the SNR of EEG generated by deep sources with weighted multielectrode leads," $J$. Physiol.-Paris 103, 306-314 (2009).

[42] Cohen, D. et. al., "MEG versus EEG localization test using implanted sources in the human brain," Ann. of Neurol. 28, 811-817 (1990).

[43] Hallez, H. et. al., "Review on solving the forward problem in EEG source analysis," J. NeuroEng. Rehab. 4:46 (2007) doi:10.1186/1743-0003-4-46.

[44] Junghöfer, M. et. al., "Mapping EEG-potentials on the surface of the brain: a strategy for uncovering cortical sources," Brain Topogr. 9, 203-217 (1997).

[45] Wolpaw, J. R. \& McFarland, D. J., "Control of a two-dimensional movement signal by a noninvasive brain-computer interface in humans," Proc. Natl. Acad. Sci. USA 101, 17849-17854 (2004).

[46] Freeman, W. J., "Use of Spatial Deconvolution to Compensate for Distortions of EEG by Volume Conduction," IEEE Trans. Biomed. Eng. 27, 421-429 (1980).

[47] Ekpar, F. E., "Method and Apparatus for Creating Interactive Virtual Tours," United States Patent 7567274 (2009).

[48] Greischar, L. L. et. al., "Effects of electrode density and electrolyte spreading in dense array electroencephalographic recording," Clin. Neurophysiol. 115, 710-720 (2004).

[49] Tenke, C. E. \& Kayser, J., "A convenient method for detecting electrolyte bridges in multichannel electroencephalogram and eventrelated potential recordings," Clin. Neurophysiol. 112, 545-550. (2001).

[50] Chang, E. F. et. al., "Categorical speech representation in human superior temporal gyrus," Nature Neuroscience 13, 1428-1432. (2010).

[51] B. Hjorth, "Source derivation simplifies topographical EEG interpretation, Am J EEG Technol 20: pp. 121-132 (1980). American Journal Of Eeg Technology (1980)

[52] C. E. Tenke, J. Kayser, "A convenient method for detecting electrolyte bridges in multichannel electroencephalogram and eventrelated potential recordings.”, Clinical Neurophysiology 112, pp. 545-550. (2001). 